\title{
Patterns of household and personal care product use by the Korean population: implications for aggregate human exposure and health risk
}

Eun Jeong Han ${ }^{1}$, KeunOh Choi ${ }^{1}$, Seunghye Sim ${ }^{1}$, Junyeong Choi ${ }^{1}$, Yoonhee Uhm², Soonbok Kim³, Eunkyung Lim² and YoungJoo Lee ${ }^{1^{*}}$

\begin{abstract}
Background: Household and personal care products (H\&PCPS) are used frequently in the domestic environment. Different types of H\&PCPs often contain the same chemicals and have potential for aggregate exposure. Therefore, product use patterns should be investigated to assess exposure and health risk.

Results: A nationwide survey was conducted in Korea to examine the use of 16 types of H\&PCPs, to obtain data that can be used for such assessments. The initial analysis focused on identifying usage patterns, such as the frequency of use and amount of product used per application. Then, co-use in 16 product categories were analyzed. Family size, marital status and age were, respectively, recognized as the critical demographic factor for classification of the frequency of use in laundry products, cleaning products and shower products. In laundry products, cleaning products and shower products, frequent H\&PCPs co-use patterns according to demographical factor were recognized. Chances of exposure of chemical ingredients to participants were investigated based on the usage of individual products. 32 chemicals among all investigated ingredients were identified to be potentially exposed to more than half of the participants, and 21 chemicals are classified above Class 4 in acute toxicity.

Conclusions: Personal preference was not observed both in the use frequency and the amount per application of H\&PCPs, among all participants. Instead, household duties were found to be associated with the yearly use frequency of household care products. In addition, the yearly chemical profiles of individual consumer were obtained, and the chemicals that have multiple chances of exposure and concomitant health risk in daily life were identified. The results provide the overview of distribution of chemicals in daily life, and essential ground for aggregate exposure assessment in Korea.
\end{abstract}

Keywords: Consumer product, Aggregate exposure, Chemical ingredient

*Correspondence: yjlee@sejong.ac.kr

1 Department of Integrated Bioscience and Biotechnology, College of Life Science, Sejong University, Kwangjingu, Kunjadong, Seoul 05006, Korea Full list of author information is available at the end of the article

\section{Background}

Consumers regularly use household and personal care products (H\&PCPs) that contain many chemicals. Some of these chemicals can irritate the skin [1], eyes [2], and respiratory tract [3]. These chemicals can also have effects harmful to health, including endocrine disruption [4], cancer [5], and pulmonary disease [6]. Fragrance 
ingredients are known to be associated with health problems $[7,8]$.

Recent studies reported that some chemical ingredients of PCPs were detected in human serum and urine, and their concentrations in human bodily fluids are related to usage of PCPs by consumers $[9,10]$. However, without usage pattern data like frequency, amount and chemical profiles, it is difficult to express the degree of risk that users may experience from the chemical ingredients of PCPs.

To determine the health risks associated with H\&PCP use, it is necessary to estimate exposure, which can be calculated from usage pattern data. Over the past 5 years, numerous studies have assessed personal care product (PCP) usage patterns [11-17], while fewer have examined household care products (HCPs) [18-20]. Many HCPs are designed for daily use, such as dishwashing agents and laundry detergents. Therefore, the chemicals in HCPs can be transferred to the body and cause adverse effects, as demonstrated for isothiazolinones $[6,21]$ and phthalates [22, 23].

Many studies of the usage patterns of consumer products have focused on the products themselves, rather than their chemical ingredients $[16,19,24-27]$. However, exposure to a product per se may not be as relevant with respect to assessing health risks. Moreover, as diverse types of products can contain the same chemicals, health hazards may arise when multiple products containing the same chemicals are used simultaneously, even when each product contains safe levels of a given chemical. Therefore, it is essential to consider the individual chemicals in consumer products when assessing usage patterns, human exposure and the corresponding health risks and to combine exposure of individual to a specific chemical via relevant routes, pathways and source [28]. The latter one, aggregate exposure assessment can be divided into product-based approach and receptor-based approach [29].

In case studies applying receptor-based approaches, self-reported data on PCP use patterns and measured concentration of ingredient were linked at an individual basis $[29,30]$. Recently, combined aggregate exposure pathway and adverse outcome pathway (AEP-AOP) approaches [31, 32] and probabilistic exposure assessments [33, 34] were developed and applied in personal care products. In these exposure assessments, PCP use data including co-use patterns is essential [35].

This study focused on co-occurring chemicals present in individual surrounding, to provide directions and guideline for consumer aggregate exposure and health risks analysis in Korea. A web-based survey was conducted on the use of 16 types of H\&PCPs. In total, 413 products were studied: $222 \mathrm{HCPs}$ and 191 PCPs.
The survey collected usage pattern data pertaining to the (1) prevalence of use, (2) type of product (e.g., liquid, powder, or spray), (3) frequency of use, (4) duration of use, and (5) amount used per application of individual products. Co-use patterns and correlations of usage patterns among product categories were analyzed based on the data for individual products. Then, the chemical ingredients in the H\&PCPs were explored, and chemicals with high exposure potential, i.e., those present in multiple product types, were identified. Finally, the health effects of the chemicals were investigated, and those known to pose hazard were identified.

\section{Materials and methods Study population}

One thousand participants were recruited from 17 metropolitan and rural areas in Korea. The study population included individuals aged between 19 and 65 years. The number of participants drawn from each province was based on the population of that province relative to the total population of Korea [36].

\section{Data collection}

A web-based questionnaire with both open and closed questions was developed and distributed in July 2018 (see Additional file 1: survey questionnaire). This survey was to analyze the personalized usage patterns of household and personal care products on an individual basis. The questionnaire enquired about the demographic characteristics of the respondents and their H\&PCP usage patterns. The demographic factors included gender, age, region of residence, income, family size, and occupation. The H\&PCP categories included laundry detergents, fabric softeners, bleaching agents, washer and laundry room cleaners, shampoos, rinses, body cleansers, mouthwashes, toothpastes, bathroom and toilet cleaners, antibacterial disinfectants, air fresheners/ deodorizers, hand sanitizers, dishwashing detergents, allpurpose cleaners, and glass and metal cleaners. Those 16 categories were selected from the most frequently used H\&PCPs in our prior survey that was conducted to select $H \& P C P$ categories and priorities that consumers use a lot. Leave-on products such as perfumes, deodorants, lotions and so on were not considered.

For each product category, the prevalence of use was determined using questions like "Which of the products below do you use in the laundry room?", where for each product type there were questions for all locations of interest. In their responses, participants could select from among products named in the questionnaire, or manually enter the name of a different product. For each product type, questions on frequency and duration of use (min, second), and amount used per application (g/ 
use), were followed. The frequency of use was recorded per day, week, or month. For each HCP category, the use of protective gloves or a mask was noted. Next, questions on the estimated amount of each product used per application were presented, with amounts recorded in milliliters $(\mathrm{mL})$ for liquids, grams $(\mathrm{g})$ for powders, counts for dispenser-type products, number of sprays for trigger-type sprays, and duration of spraying for aerosoltype sprays. A shot glass $(50 \mathrm{~mL})$, paper cup $(180 \mathrm{~mL})$, and tablespoon $(10 \mathrm{~g})$ were provided as example amounts. The 111 products among the various H\&PCP categories were selected in order of use by respondents and purchased. In the laboratory, we measured the weight ( $\mathrm{g}$ ) of product dispensed per spray for trigger-type sprays, that of aerosol spray applied per unit time, and that of liquid products per cup of liquid products. For products with no laboratory data, the amount used per application (g/use) was estimated based on available data for other products of that type.

The participants reported using 413 different products, the ingredients of which were analyzed. We searched webpages of makers and labels of products to determine the ingredients in the products; 690 ingredients were identified in all.

\section{Data analysis}

All statistical analyses were conducted using $\mathrm{R}$ software (ver. 3.6.0; R Development Core Team, Vienna, Austria).

\section{Product usage patterns}

Product usage patterns were estimated using questions regarding the frequency of use and amount of product used per application. The yearly frequency of use (use/ year) was calculated based on the reported daily, weekly, or monthly use. The median amount per application (g) was calculated for each respondent based on the use data for all H\&PCPs. Decision tree classification was used to analyze usage patterns for each product category according to demographic factors such as gender, age group, marital status, and family size. A $p$-value $<0.05$ indicated statistical significance, unless stated otherwise.

\section{Co-use of products}

The co-use of consumer products that contain the same chemicals results in aggregate exposure. The co-use of products from different categories within each use purpose (i.e., laundry, cleaning and shower) was assessed. Then, the percentage of respondents indicating to use the products according to each co-use combination, and the cumulative sum of the fractions, were calculated. Spearman's correlation coefficient $(R)$ was used to measure the strength of correlations between the H\&PCPs in each category, in terms of frequency and amount used.

\section{Results and discussion \\ Demographics of the study population}

Table 1 summarizes the demographics of the study population, which was $51.0 \%$ female and $49.0 \%$ male. For the analysis, the participants were divided into five age groups: 20s (19-29 years), 30s (30-39 years), 40s (40-49 years), 50s (50-59 years), and $60+$ years. These groups contained 20.8, 20.5,24.2, 23.6, and $10.9 \%$ of the participants, respectively. The regions presented in Table 1 are all the administrative districts in Korea. The demographics of the participants in each province, gender, and age subgroup were in accordance with nationwide norms for 2016 [36-38] (Table 1).

\section{Product usage patterns}

The daily use frequency (use/day) and the amount used per application (g/use) for various demographic groups of the study population are summarized in Additional file 2: Tables S1 and S2, respectively. For international relevance, use frequency values for shower products were compared with those in Scientific Committee on Consumer Safety (SCCS) guidance [39] and a French study [25] (Table 2). The range of use frequency values of shampoo, rinse and body cleanser in this study $(0.78$ $1.46,0.62-0.95$, and $0.73-1.14$ per day, respectively; from all demographic groups) were comparable to those of French population $(0.44-0.67,0.37-0.47$, and $0.87-1.04$

\begin{tabular}{|c|c|c|c|c|}
\hline \multirow[t]{2}{*}{ Groups } & \multicolumn{2}{|l|}{ Survey } & \multicolumn{2}{|c|}{ National statistics } \\
\hline & (Person) & $(\%)$ & (Person) & (\%) \\
\hline \multicolumn{5}{|l|}{ Gender } \\
\hline Male & 510 & 51.0 & $25,768,055$ & 50.1 \\
\hline Female & 490 & 49.0 & $25,654,452$ & 49.9 \\
\hline \multicolumn{5}{|l|}{ Age } \\
\hline $20 \mathrm{~s}$ & 208 & 20.8 & $6,925,447$ & 19.7 \\
\hline $30 \mathrm{~s}$ & 205 & 20.5 & $7,518,454$ & 21.4 \\
\hline $40 \mathrm{~s}$ & 242 & 24.2 & $8,570,005$ & 24.3 \\
\hline $50 \mathrm{~s}$ & 236 & 23.6 & $8,380,783$ & 23.8 \\
\hline Over 60 & 109 & 10.9 & $3,812,645$ & 10.8 \\
\hline \multicolumn{5}{|l|}{ Family size } \\
\hline 1 & 84 & 8.4 & $5,618,677$ & 28.6 \\
\hline 2 & 198 & 19.8 & $5,260,332$ & 26.7 \\
\hline 3 & 285 & 28.5 & $4,178,641$ & 21.2 \\
\hline 4 & 358 & 35.8 & $3,473,897$ & 17.7 \\
\hline Over 5 & 75 & 7.5 & $1,142,328$ & 5.8 \\
\hline
\end{tabular}


Table 2 Comparison of daily frequency values among this study and studies for French and SCCS (use/day)

\begin{tabular}{|c|c|c|c|c|c|}
\hline & \multicolumn{2}{|l|}{ Korea $^{a}$} & \multicolumn{2}{|l|}{ French $^{\mathbf{b}}$} & \multirow[t]{2}{*}{$\operatorname{sCCS}^{c}$} \\
\hline & Mean $^{d}$ & P95 & Mean $^{d}$ & P95 & \\
\hline Laundry detergent & $0.28-0.75$ & 1.0 & - & - & - \\
\hline Fabric softener & $0.29-0.61$ & 1.0 & - & - & - \\
\hline Bleaching agent & $0.1-0.28$ & 1.0 & - & - & - \\
\hline $\begin{array}{l}\text { Washer and laundry room } \\
\text { cleaner }\end{array}$ & $0.08-0.19$ & 0.4 & - & - & - \\
\hline Shampoo & $0.78-1.46$ & 2.0 & $0.44-0.67$ & $1-2$ & 1 \\
\hline Rinse $^{e}$ & $0.62-0.95$ & 2.0 & $0.37-0.47$ & 1 & 0.28 \\
\hline Body cleanser ${ }^{f}$ & $0.73-1.14$ & 2.0 & $0.87-1.04$ & 2 & 1.43 \\
\hline Mouthwash & $0.56-1.03$ & 3.0 & - & - & - \\
\hline Toothpaste & $2.28-2.69$ & 4.0 & - & - & - \\
\hline Bathroom and toilet cleaner & $0.18-0.41$ & 1.0 & - & - & - \\
\hline Disinfectant & $0.11-0.22$ & 0.4 & - & - & - \\
\hline Air freshener/deodorizer & $0.4-0.79$ & 2.0 & - & - & - \\
\hline Hand sanitizer & $0.51-1.13$ & 5.0 & - & - & - \\
\hline Dishwashing detergent & $1.27-2.54$ & 5.0 & - & - & - \\
\hline All-purpose cleaner & $0.12-0.3$ & 1.0 & - & - & - \\
\hline Glass and metal cleaner & $0.03-0.07$ & 0.3 & - & - & - \\
\hline
\end{tabular}

a This study

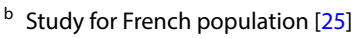

c Scientific Committee on Consumer Safety (SCCS) guidance [39]

d Values obtained from the various subpopulations (gender, marital status, family size, age group for Korean; adult women, adult pregnant women, adult men, girls, boys, baby girls and baby boys for French)

e Hair conditioner in European studies

${ }^{f}$ Shower gel in European studies

per day, respectively; from all demographic groups), and to values in SCCS guidance (1, 0.28, and 1.43 per day, respectively).

The yearly use frequency (use/year), for every respondent and for all 413 H\&PCPs, was classified according to demographic factors such as gender, age group, marital status, family size, monthly income, and region of residence using decision trees (ctree function in partykit package of $\mathrm{R}$ ).

Figure 1 shows the decision trees for the yearly use frequency of ten selected H\&PCP categories. For six H\&PCP categories (mouthwash, toothpaste, air freshener/deodorizer, all-purpose cleaner, glass and metal cleaner, hand sanitizer), remarkable classification was not observed.
Figure 1a-c shows, respectively, the distribution of respondents according to the yearly frequency of usage of laundry detergent, fabric softener and bleaching agent, categorized as low (LF; 0-156/year), medium (MF; 157-365/year), or high (HF; 366+/year) frequency. In cases of laundry products, the number of family members (that is to say, the amount of clothes to be washed) was important, where the yearly use frequency of the subjects with families comprising less than two people was mostly LF.

Figure $1 \mathrm{~d}-\mathrm{g}$ shows, respectively, the distribution of respondents according to the yearly frequency of usage of washer and laundry room cleaner, bathroom and toilet cleaner, disinfectant and dishwashing detergent. In cases of cleaning products, the marital status (Fig. 1d, f, g) and the gender (Fig. 1d, e, g) were important, where the yearly use frequency of the subjects who are single or male was lower than other respondents. These results indirectly indicate that married females bear a greater burden with respect to household labor than married males in Korean society.

Figure $1 \mathrm{~h}-\mathrm{j}$ shows, respectively, the distribution of respondents according to the yearly frequency of usage of shampoo, rinse and body cleanser. In cases of shower products, the age group was important, where the yearly use frequency of the subjects who are old was mostly LF. These results are possibly because commuting (to school or office) increases the frequency of showers.

To sum up, demographic factors were associated with the yearly use frequency of certain types of H\&PCPs (Fig. 1, Table 3). The amount of product used per application was not associated with demographic factors for most of the H\&PCPs.

\section{Co-use}

The co-use of products in different categories was examined (Table 3). Without sub-grouping of categories, remarkable pattern of co-use was not observed. As laundry products, cleaning products and shower products showed similar usage pattern in the demographic subgroup in "Product usage patterns" section, the co-use was examined in each group.

As shown in Table 3, in using laundry products, 73.7\% of the subjects with families comprising more than three people used all the laundry detergent, fabric softener as well as bleaching agent. In using cleaning products,

(See figure on next page.)

Fig. 1 Classification of yearly use frequencies (use/year) of selected H\&PCP categories with demographic factors. a Laundry detergent: LF (low frequency, 0-156 count/year), MF (medium frequency, 156-365 count/year), HF (high frequency, 365 count/year). b Fabric softener: LF (0-156), MF (156-365), HF (365 ). c Bleaching agent: LF (0-156), MF (156-365), HF (365 ). d Washer and laundry room cleaner: LF (0-12), MF (12-52), HF (52 ). e Bathroom and toilet cleaner: LF (0-12), MF (12-52), HF (52 ). f Disinfectant: LF (0-12), MF (12-52), HF (52 ). g Dishwashing detergent: LF (0-365), MF (365-730), HF (730 ). h Shampoo: LF (0-365), MF (365-730), HF (730 ). i Rinse: LF (0-365), MF (365-730), HF (730 ). j Body cleanser: LF (0-365), MF (365-730), HF (730 ) 


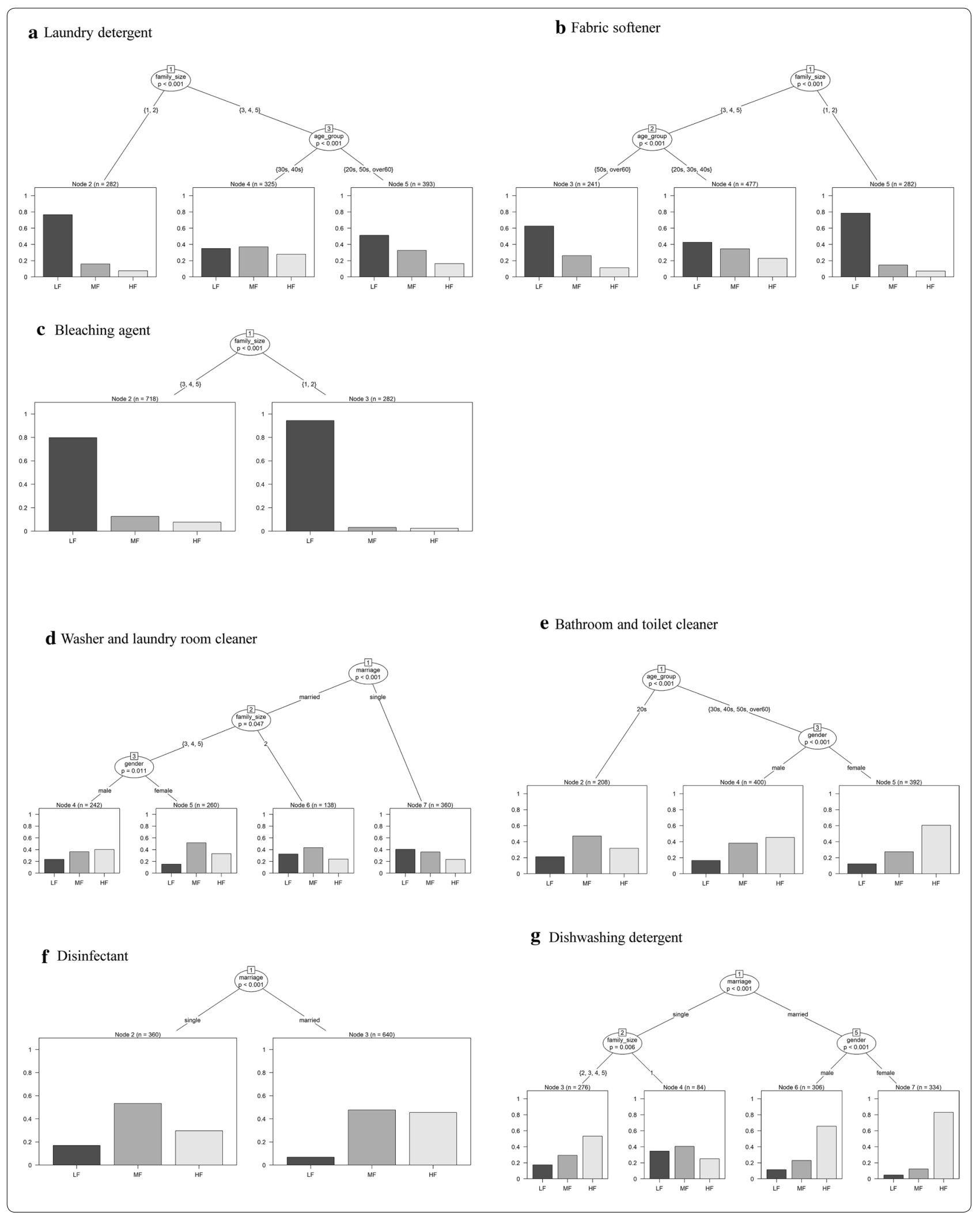




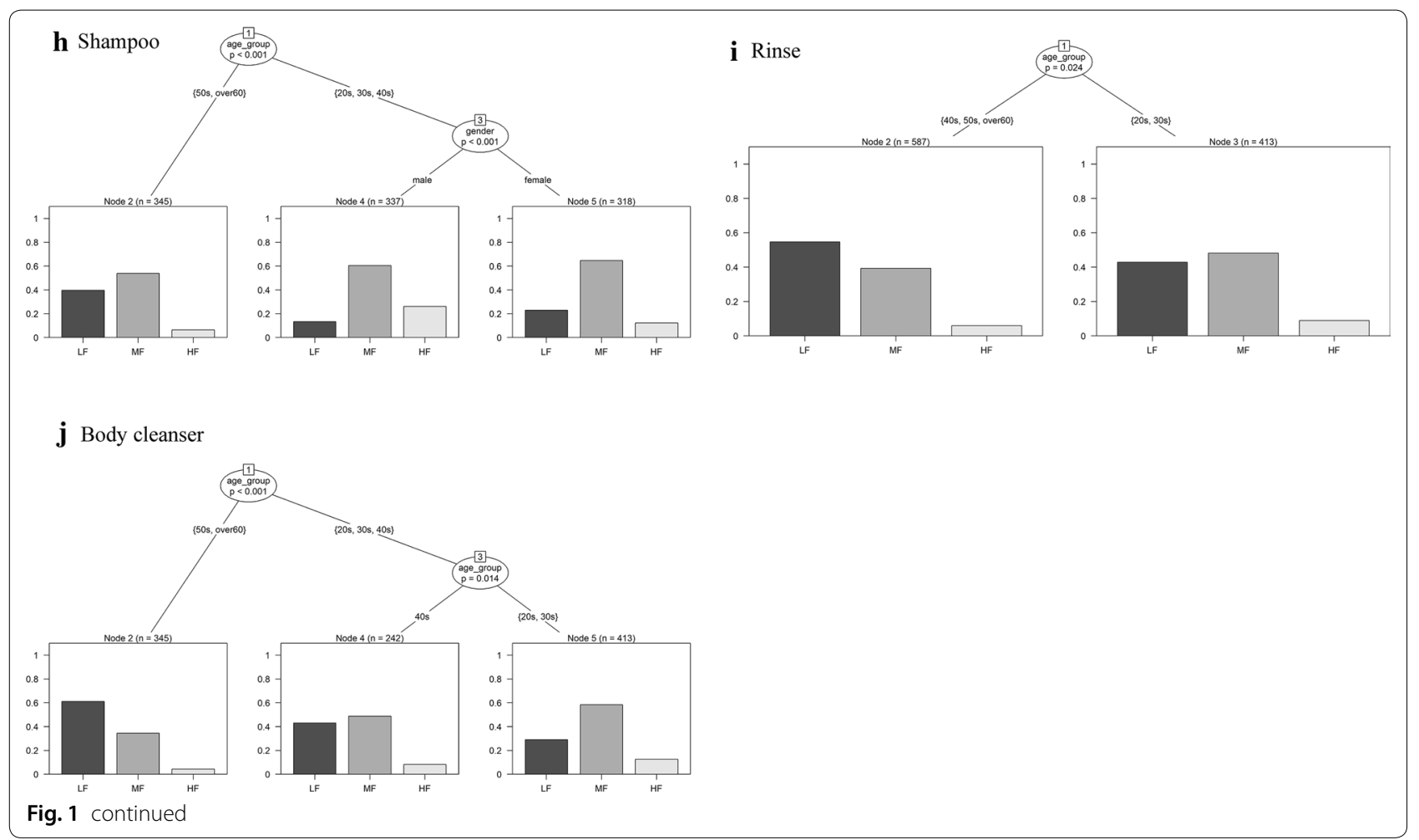

Table 3 Three most frequent H\&PCP co-use combinations in each usage

\begin{tabular}{|c|c|c|c|}
\hline Usage & Group & Co-use combinations & Users (\%) \\
\hline \multirow[t]{6}{*}{ Laundry } & Family size under 2 & G01, G02, G03 & 64.9 \\
\hline & & G01, G02 & 24.8 \\
\hline & & G01 & 5.0 \\
\hline & Family size over 3 & G01, G02, G03 & 73.7 \\
\hline & & $\mathrm{G} 01, \mathrm{G} 02$ & 19.4 \\
\hline & & G01, G03 & 3.3 \\
\hline \multirow[t]{6}{*}{ Cleaning } & Single & G04, G10, G11, G14 & 52.2 \\
\hline & & G10, G11, G14 & 18.1 \\
\hline & & G11, G14 & 8.6 \\
\hline & Married & G04, G10, G11, G14 & 70.5 \\
\hline & & G10, G11, G14 & 11.4 \\
\hline & & G11, G14 & 7.2 \\
\hline \multirow[t]{6}{*}{ Shower } & Age $<50$ & G05, G06, G07 & 77.4 \\
\hline & & G05, G07 & 18.5 \\
\hline & & G05 & 2.1 \\
\hline & Age $\geq 50$ & G05, G06, G07 & 73.0 \\
\hline & & G05, G07 & 17.1 \\
\hline & & G05, G06 & 5.5 \\
\hline
\end{tabular}

G01: laundry detergent, G02: fabric softener, G03: bleaching agent, G04: washer and laundry room cleaner, G05: shampoo, G06: rinse, G07: body cleanser, G08: mouthwash, G09: toothpaste, G10: bathroom and toilet cleaner, G11: disinfectant, G12: air freshener/deodorizer, G13: hand sanitizer, G14: dishwashing detergent, G15: all-purpose cleaner, G16: glass and metal cleaner
$70.5 \%$ of the married subjects the washer and laundry room cleaner, bathroom and toilet cleaner, as well as disinfectant and dishwashing detergents. In using shower products, $77.4 \%$ of the subjects of ages over 50 the shampoo, rinse as well as body cleanser.

\section{Correlation of usage patterns between pairs of H\&PCP categories}

It may be expected that an individual who used a product from a given category frequently would also use a product from other categories frequently. Therefore, we determined Spearman's correlations $(R)$ between all pairs of H\&PCP categories, for yearly use frequency and amount of product used per application. Correlations were classified as very weak $(0<R<0.19)$, weak $(0.20<R<0.39)$, moderate $(0.40<R<0.59)$, strong $(0.60<R<0.79)$, or very strong $(0.80<R<1.00)$. A $p$-value of $<0.05$ indicated statistical significance unless noted otherwise.

First, Spearman's $R$ was calculated for the yearly use frequencies of all H\&PCPs within each product category (see Additional file 2: Table S3). The correlations ranged from very weak to moderate $(R=0.08-0.50)$, except for the strong correlation between laundry detergent and fabric softener $(R=0.77, p<0.01)$. This indicates no clear trends in yearly use frequencies of products from different categories. 


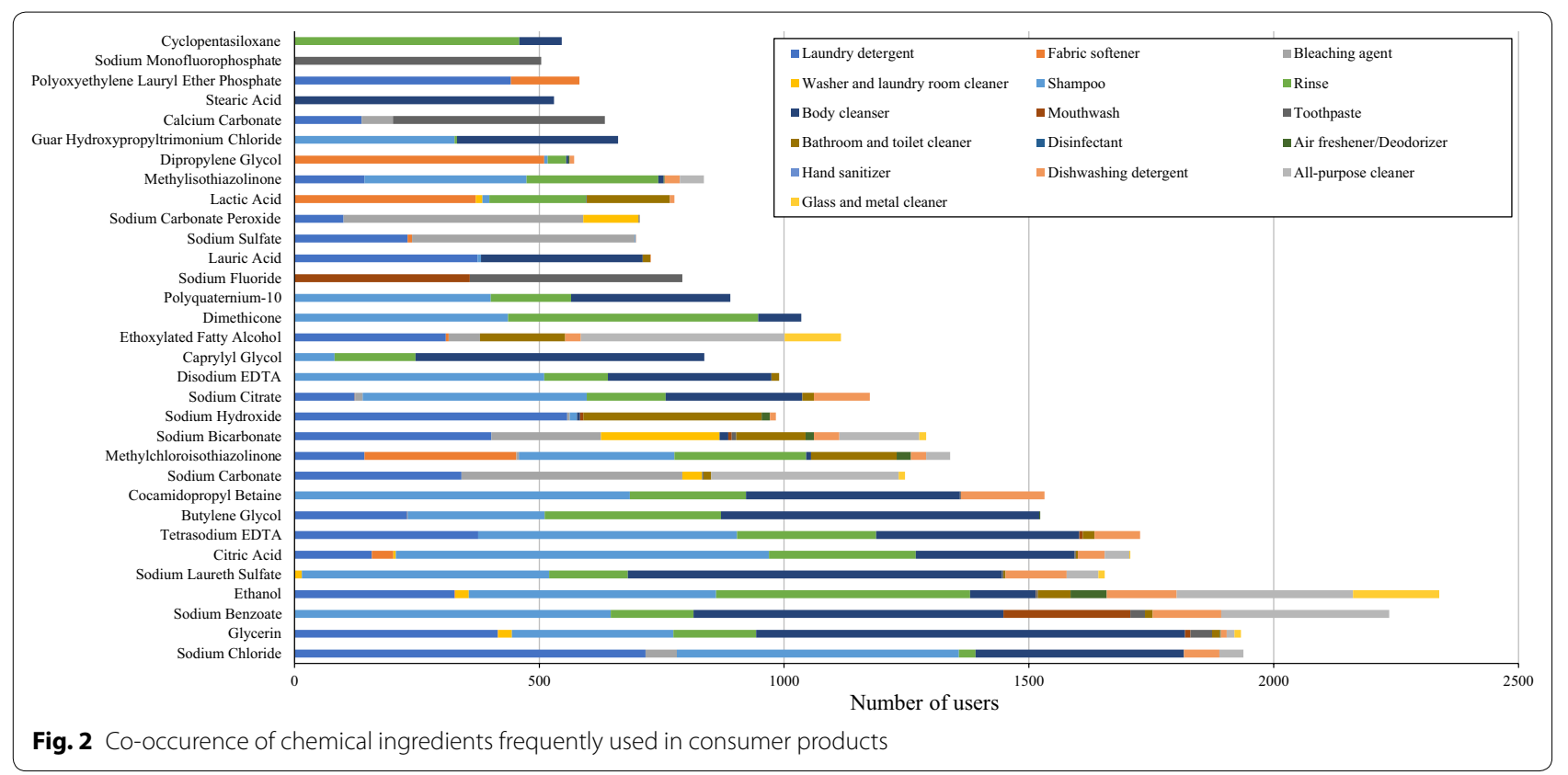

Spearman's $R$ was then calculated for the amount of product used per application, for all H\&PCPs within each product category (Additional file 2: Table S4). The correlations ranged from very weak to moderately positive $(R=0.07-0.58)$. The strongest correlation was observed between shampoo and body cleanser $(R=0.58, p<0.01)$, and the second strongest was that between shampoo and rinses $(R=0.55, p<0.01)$. There were no pairs of H\&PCPs with strong or very strong correlations. This indicates no clear trends at the individual level in amount of product used per application among the H\&PCP types considered in this study. Personal preference was not observed both in the use frequency and the amount per application of H\&PCPs, among all participants.

\section{Chemicals of concern}

The chemical ingredients of 413 products indicated by the respondents were investigated. Across all products, there were 690 chemicals that users can be exposed to. We calculated the number of respondents exposed to the chemicals in each H\&PCP category over the course of the survey (Additional file 2: Table S5). For example, 717 respondents were exposed to sodium chloride in laundry detergents, and 64 to sodium chloride in bleaching agents. Because of the wide variability in the chemical ingredients, we extracted 32 chemicals that more than half of the participants could be exposed to and evaluated their prevalence across the 16 product categories using a stacked bar chart (Fig. 2).
The hazard classifications of the Global Harmonized System (GHS) for 32 chemicals are listed in Additional file 2: Table S6; most of chemicals (except ethoxylated fatty alcohol, polyquaternium-10) are classified to Class 2 in eye irritation. 21 chemicals are classified above Class 4 in acute toxicity, and especially 3 chemicals (methylchloroisothiazolinone, dimethicone, methylisothiazolinone) are classified to Class 1 in acute toxicity.

\section{Consumers with many chances of exposure to hazardous chemicals}

The yearly chances of exposure of every respondent to 690 chemicals was counted based on the use frequency of all products containing each chemical. Of the five respondents that used products from the highest number of categories (14 categories), one respondent who had chances of exposure to the greatest number of chemicals (103 chemicals) was selected and analyzed with respect to annual chances of exposure to the various chemicals (Additional file 2: Table S7). This respondent exemplifies a consumer with many chances of exposure to chemicals as a result of using many H\&PCPs. For example, the results in Additional file 2: Table S7 indicate that this individual could be exposed to tetrasodium EDTA 365 times/year via shampoo, 365 times/year via rinses, 365 times/year via body cleansers, and 1095 times/year via dishwashing detergents, giving a total of 2190 times/year. Of the 103 chemicals listed in Additional file 2: Table S7, the individual could be exposed to 24 chemicals more than 730 times/year (i.e., 2 times/day on average). We 


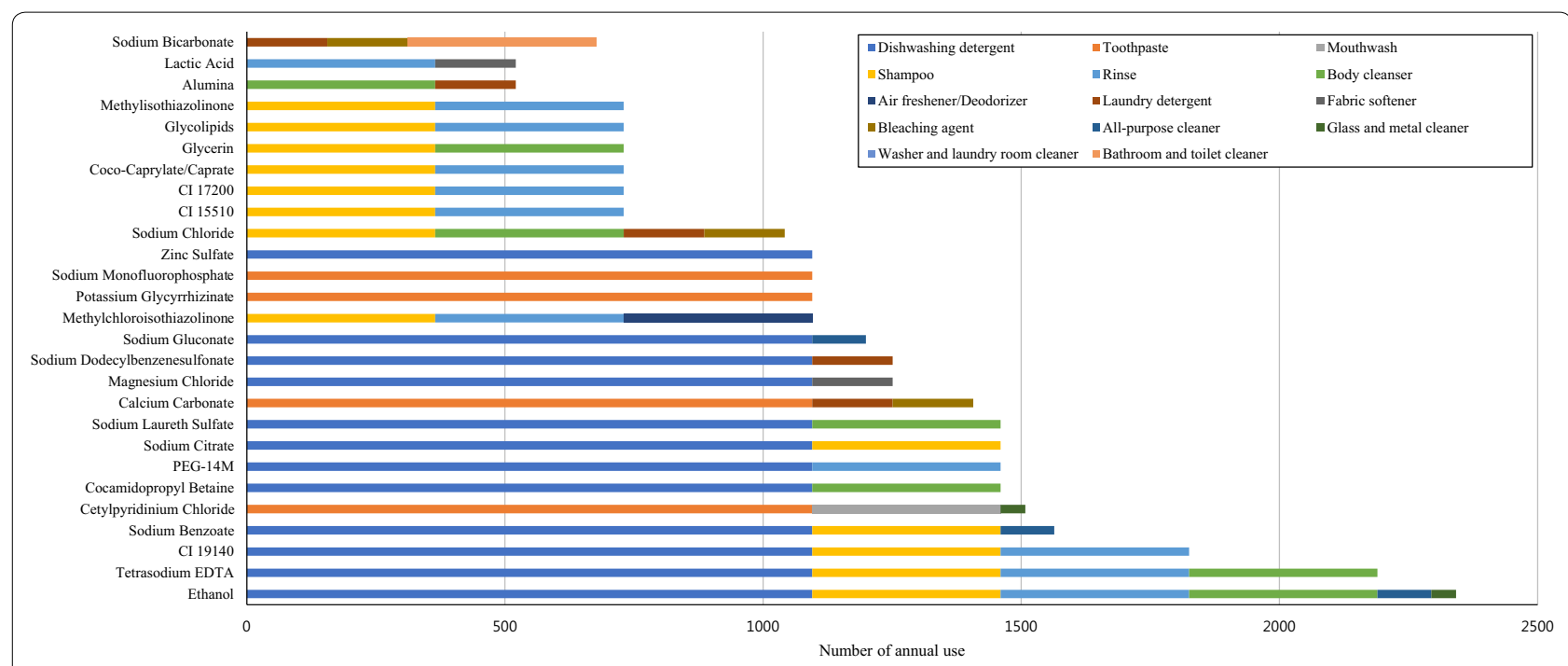

Fig. 3 Co-occurence of chemical ingredients frequently used by one selected respondent who used most various chemicals

evaluated the prevalence of these chemicals across the 14 product categories using a stacked bar chart (Fig. 3).

The hazard classifications of the GHS for the 24 chemicals mentioned above are listed in Additional file 2: Table S8. Most of chemicals (except sodium gluconate, potassium glycyrrhizinate, coco-caprylate/caprate and glycolipids) are classified above Class 4 in health hazards.

\section{Limitations}

There were several limitations to this study. First, the study population of 1000 was not large enough to be representative of the total population of Korea. However, as shown in Table 1, the demographics of the participants in each province, gender, and age group were in accordance with nationwide norms [36-38], so the sample was appropriate for a screening-level analysis. Nevertheless, this limitation would be ameliorated if the sample size was increased, which we plan to do for our next survey.

Second, data on the product usage patterns were collected using a web-based questionnaire; this might exclude people who cannot access the Internet, but use $\mathrm{H} \& \mathrm{PCPs}$ frequently or heavily. However, in Korea, the Internet usage rate is $99.9 \%$ for those in their $20 \mathrm{~s}$ and $30 \mathrm{~s}$, 99.7\% for those in their 40 s, $98.7 \%$ for those in their 50 s, and $88.8 \%$ for those in their 60 s [40], so an online survey can cover most of the Korean population in practice.

Third, this study collected data only on the current habits and behaviors of the participants. Therefore, the data might be biased toward products used in the summer (i.e., July). Rates of household and personal cleaning are usually higher in summer than in winter, so the yearly usage frequencies and amounts used per application in this study might have been overestimated.

Fourth, this study relied on self-reported data. Though the detail guide was supplied in survey, it is difficult to expect that all participants reported data consistently. And those self-reported data were not checked in house of participants.

Finally, some major brand names in each product group were suggested in the survey, to provide answering convenience for the participants. This could create a bias towards the answers suggested, but the bias may not be so problematic in our survey. For example, we suggested 16 brand names for shampoo, but respondents provided 58 brand names.

\section{Conclusion}

In this study, a survey was distributed nationwide to assess the use of H\&PCPs by Koreans, to generate consumer usage pattern data to inform exposure and risk assessments. This survey captured information on the prevalence, frequency, and amount of use of 16 types of H\&PCPs, in addition to demographic factors associated with the use of the products. Family size, marital status and age were, respectively, recognized as the critical demographic factor for classification of the frequency of use in laundry products, cleaning products and shower products. Clear trends in usage habits at the individual level, with respect to use frequency and amount, were lacking. Certain chemicals in many of the studied products have many chances of exposure to person and concomitant health risks. In addition, consumers who 
co-use various H\&PCPs can be exposed to many chemicals, some of which has hazard to health. As mentioned above, the sample size and cross-sectional nature of the survey represent limitations, so further studies including more subjects and a longitudinal component are recommended. The results of this study may serve as an important resource for assessing the potential aggregate exposure and health risks of various H\&PCPs.

\section{Supplementary information}

Supplementary information accompanies this paper at https://doi. org/10.1186/s12302-020-00417-3.

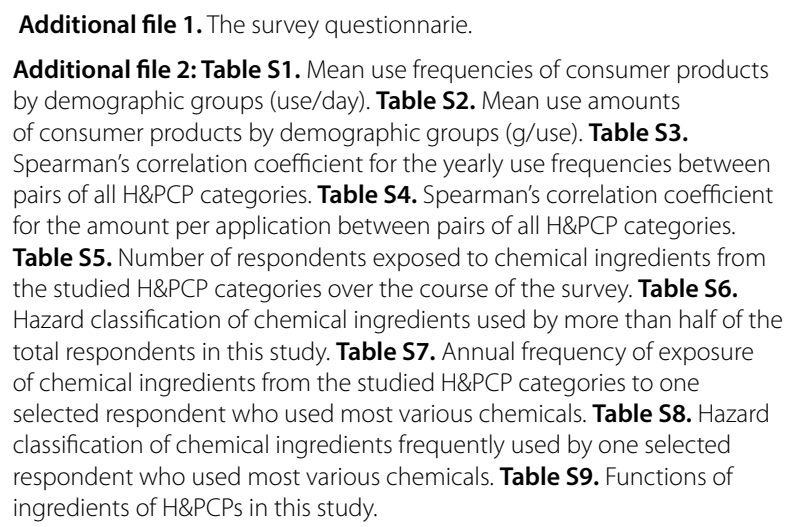

Additional file 2: Table S1. Mean use frequencies of consumer products by demographic groups (use/day). Table S2. Mean use amounts of consumer products by demographic groups (g/use). Table S3. Spearman's correlation coefficient for the yearly use frequencies between pairs of all H\&PCP categories. Table S4. Spearman's correlation coefficient for the amount per application between pairs of all H\&PCP categories. Table S5. Number of respondents exposed to chemical ingredients from the studied H\&PCP categories over the course of the survey. Table S6. Hazard classification of chemical ingredients used by more than half of the total respondents in this study. Table S7. Annual frequency of exposure of chemical ingredients from the studied H\&PCP categories to one selected respondent who used most various chemicals. Table S8. Hazard classification of chemical ingredients frequently used by one selected respondent who used most various chemicals. Table S9. Functions of ingredients of H\&PCPs in this study.

\section{Abbreviations}

H\&PCPs: Household and personal care products; HCPs: Household care products; PCPs: Personal care products; GHS: Global Harmonized System; SCCS: Scientific Committee on Consumer Safety; LF: Low yearly frequency of usage; MF: Medium yearly frequency of usage; HF: High yearly frequency of usage.

\section{Acknowledgements}

Not applicable.

\section{Authors' contributions}

YJL, EL, SK, YU designed and developed the basic ideas of the study. EJH, KOC, SS, JC performed the data analysis. EJH was a major contributor in writing the manuscript. All authors read and approved the final manuscript.

\section{Funding}

This research was supported by Research Program to Solve Social Issues of the National Research Foundation of Korea (NRF) funded by the Ministry of Science and ICT (No. NRF-2017M3C8A6091777) and by Korea Ministry of Environment (MOE) as Graduate School specialized in Climate Change.

\section{Availability of data and materials}

Not applicable.

\section{Ethics approval and consent to participate}

Not applicable.

\section{Consent for publication}

Not applicable.

\section{Competing interests}

The authors declare that they have no known competing financial interests or personal relationships that could have appeared to influence the work reported in this paper.

\section{Author details}

${ }^{1}$ Department of Integrated Bioscience and Biotechnology, College of Life Science, Sejong University, Kwangjingu, Kunjadong, Seoul 05006, Korea.

2 Korea National Council of Consumer Organizations, SEOUL YWCA BId. \#701, 20, Myungdong 11 gil, Jung-gu, Seoul, Korea. ${ }^{3}$ Korean Woman's Federation for Consumer, Sangdong Bld. \#603, Namdaemun-ro 30, Jung-gu, Seoul, Korea.

Received: 7 February 2020 Accepted: 26 September 2020

Published online: 08 October 2020

\section{References}

1. Basketter DA, Lemoine S, McFadden JP (2015) Skin sensitisation to fragrance ingredients: is there a role for household cleaning/maintenance products? Eur J Dermatol 25:7-13. https://doi.org/10.1684/ejd.2014.2472

2. Lim DS, Roh TH, Kim MK, Kwon YC, Choi SM, Kwack SJ, Kim KB, Yoon S, Kim HS, Lee BM (2018) Non-cancer, cancer, and dermal sensitization risk assessment of heavy metals in cosmetics. J Toxicol Environ Health $A$ 81:432-452. https://doi.org/10.1080/15287394.2018.1451191

3. Burg W, Bouma K, Schakel DJ, Wijnhoven SWP, Engelen J, Loveren H, Ezendam J (2014) Assessment of the risk of respiratory sensitization from fragrance allergens released by air fresheners. Inhal Toxicol 26:310-318. https://doi.org/10.3109/08958378.2014.888110

4. Giulivo M, Alda ML, Capri E, Barceló D (2016) Human exposure to endocrine disrupting compounds: their role in reproductive systems, metabolic syndrome and breast cancer. A review. Environ Res 151:251-264. https://doi.org/10.1016/j.envres.2016.07.011

5. Lefebvre MA, Meuling WJA, Engel R, Coroama MC, Renner G, Pape W, Nohyne GJ (2012) Consumer inhalation exposure to formaldehyde from the use of personal care products/cosmetics. Regul Toxicol Pharmacol 63:171-176. https://doi.org/10.1016/j.yrtph.2012.02.011

6. Lee JH, Yu IJ (2017) Human exposure to polyhexamethylene guanidine phosphate from humidifiers in residential settings: cause of serious lung disease. Toxicol Ind Health 33:835-842. https://doi.org/10.1177/07482 33717724983

7. Steinemann A (2019) International prevalence of fragrance sensitivity. Air Qual Atmos Health 12:891-897. https://doi.org/10.1007/s11869-01900699-4

8. Wieck S, Olsson O, Kümmerer K, Klaschka U (2018) Fragrance allergens in household detergents. Regul Toxicol Pharmacol 97:163-169. https://doi. org/10.1016/j.yrtph.2018.06.015

9. Buckley JP, Palmieri RT, Matuszewski JM, Herring AH, Baird DD, Hartmann KE, Hoppin JA (2012) Consumer product exposures associated with urinary phthalate levels in pregnant women. J Expo Sci Environ Epidemiol 22:468-475. https://doi.org/10.1038/jes.2012.33

10. Philippat C, Bennett D, Calafat AM, Picciotto IH (2015) Exposure to select phthalates and phenols through use of personal care products among Californian adults and their children. Environ Res 140:369-376. https:// doi.org/10.1016/j.envres.2015.04.009

11. Bernard A, Dornic N, Roudot A, Ficheux A (2018) Probabilistic exposure assessment to face and oral care cosmetic products by the French population. Food Chem Toxicol 111:511-524. https://doi.org/10.1016/j. fct.2017.11.056

12. Comiskey D, Api AM, Barratt C, Daly EJ, Ellis G, McNamara C, O'Mahony C, Robison SH, Safford B, Smith B, Tozer S (2015) Novel database for exposure to fragrance ingredients in cosmetics and personal care products. Regul Toxicol Pharmacol 72:660-672. https://doi.org/10.1016/j.yrtph 2015.05.012

13. Comiskey D, Api AM, Barratt C, Ellis G, McNamara C, O'Mahony C, Robison SH, Rose J, Safford B, Smith B, Tozer S (2017) Integrating habits and practices data for soaps, cosmetics and air care products into an existing aggregate exposure model. Regul Toxicol Pharmacol 88:144-156. https:// doi.org/10.1016/j.yrtph.2017.05.017

14. Gomez-Berrad MP, Ficheux AS, Rakotomalala S, Guillou S, Bellec M, Javel DD, Roudot AC, Ferret PJ (2018) Consumption and exposure assessment to sunscreen products: a key point for safety assessment. Food Chem Toxicol 114:170-179. https://doi.org/10.1016/j.fct.2018.02.035

15. Jung K, Choi M, Hong S, Park B, Park I, Shin J, Lee T, Park GH, Kim M (2018) Realistic and aggregated exposure assessment of Korean men and 
women to color make-up products. Food Chem Toxicol 118:382-389. https://doi.org/10.1016/j.fct.2018.05.020

16. Park GH, Nam C, Hong S, Park B, Kim H, Lee T, Kim K, Lee JH, Kim MH (2018) Socioeconomic factors influencing cosmetic usage patterns. J Expo Sci Environ Epidemiol 28:242-250. https://doi.org/10.1038/ jes.2017.20

17. Safford B, Api AM, Barratt C, Comiskey D, Daly EJ, Ellis G, McNamara C, O'Mahony C, Robison S, Smith B, Thomas R, Tozer S (2015) Use of an aggregate exposure model to estimate consumer exposure to fragrance ingredients in personal care and cosmetic products. Regul Toxicol Pharmacol 72:673-682. https://doi.org/10.1016/j.yrtph.2015.05.017

18. Garcia-Hidalgo E, Goetz N, Siegrist M, Hungerbühler K (2017) Use-patterns of personal care and household cleaning products in Switzerland. Food Chem Toxicol 99:24-39. https://doi.org/10.1016/j.fct.2016.10.030

19. Park JY, Lee K, Hwang Y, Kim JH (2015) Determining the exposure factors of personal and home care products for exposure assessment. Food Chem Toxicol 77:105-110. https://doi.org/10.1016/j.fct.2015.01.002

20. Zaleski RT, Egeghy PP, Hakkinen PJ (2016) Exploring global exposure factors resources for use in consumer exposure assessments. Int J Environ Res Public Health 13:1-26. https://doi.org/10.3390/ijerph13070744

21. Garcia-Hidalgo E, Schneider D, Goetz N, Delmaar C, Siegrist M, Hungerbühler K (2018) Aggregate consumer exposure to isothiazolinones via household care and personal care products: probabilistic modelling and benzisothiazolinone risk assessment. Environ Int 118:245-256. https://doi. org/10.1016/j.envint.2018.05.047

22. Jaakkola JJK, Knight TL (2008) The role of exposure to phthalates from polyvinyl chloride products in the development of asthma and allergies: a systematic review and meta-analysis. Environ Health Perspect 116:845-853. https://doi.org/10.1289/ehp.10846

23. Marsee K, Woodruff TJ, Axelrad DA, Calafat AM, Swan SH (2006) Estimated daily phthalate exposures in a population of mothers of male infants exhibiting reduced anogenital distance. Environ Health Perspect 114:805-809. https://doi.org/10.1289/ehp.8663

24. Biesterbos JWH, Dudzina T, Delmaar CJE, Bakker MI, Russel FGM, Goetz N, Scheepers PTJ, Roeleveld N (2013) Usage patterns of personal care products: important factors for exposure assessment. Food Chem Toxicol 55:8-17. https://doi.org/10.1016/j.fct.2012.11.014

25. Ficheux AS, Wesolek N, Chevillotte G, Roudot AC (2015) Consumption of cosmetic products by the French population. First part: frequency data. Food Chem Toxicol 78:159-169. https://doi.org/10.1016/j.fct.2015.01.016

26. Gomez-Berrada MP, Ficheux AS, Guillou S, Berge C, Javel D, Roudot AC, Ferret PJ (2017) Consumption and exposure assessment to cosmetic products for children under 2 years old. Food Chem Toxicol 105:151-160. https://doi.org/10.1016/j.fct.2017.04.011

27. Park JY, Lim M, Yang W, Lee K (2017) Exposure factors for cleaning, automotive care, and surface protection products for exposure assessments. Food Chem Toxicol 99:128-134. https://doi.org/10.1016/j.fct.2016.11.024

28. US EPA (2003) Framework for Cumulative Risk Assessment. U.S. Environmental Protection Agency, Office of Research and Development, Center for Public Health and Environmental Assessment (CPHEA), formerly known as the National Center for Environmental Assessment (NCEA), Washington Office, Washington, DC, EPA/600/P-02/001F

29. Lim M, Park JY, Lim JE, Moon HB, Lee K (2019) Receptor-based aggregate exposure assessment of phthalates based on individual's simultaneous use of multiple cosmetic products. Food Chem Toxicol 127:163-172. https://doi.org/10.1016/j.fct.2019.03.031

30. Manová E, Goetz N, Hungerbuehler K (2015) Aggregate consumer exposure to UV filter ethylhexyl methoxycinnamate via personal care products. Environ Int 74:249-257. https://doi.org/10.1016/j.envint.2014.09.008
31. Hines DE, Conolly RB, Jarabek AM (2019) A quantitative source-tooutcome case study to demonstrate the integration of human health and ecological end points using the aggregate exposure pathway and adverse outcome pathway frameworks. Environ Sci Technol 53:1 100211012. https://doi.org/10.1021/acs.est.9b04639

32. Clewell RA, Leonard JA, Nicolas Cl, Campbell JL, Yoon M, Efremenko AY, McMullen PD, Andersen ME, Clewell HJ III, Phillips KA, Tan YM (2020) Application of a combined aggregate exposure pathway and adverse outcome pathway (AEP-AOP) approach to inform a cumulative risk assessment: a case study with phthalates. Toxicol Vitro. https://doi. org/10.1016/j.tiv.2020.104855

33. Karrer C, Boer W, Delmaar C, Cai Y, Crépet A, Hungerbühler K, Goetz N (2019) Linking probabilistic exposure and pharmacokinetic modeling to assess the cumulative risk from the bisphenols BPA, BPS, BPF, and BPAF for Europeans. Environ Sci Technol 53:9181-9191. https://doi.org/10.1021/ acs.est.9b01749

34. Tokumura M, Nitta S, Hayashi T, Yamaguchi R, Wang Q, Miyake Y, Amagai T, Makino M (2020) Probabilistic exposure assessment of aggregate rates of dermal exposure of Japanese women and children to parabens in personal care products. Chemosphere 239:124704. https://doi.org/10.1016/j. chemosphere.2019.124704

35. Manová E, Goetz N, Keller C, Siegrist M, Hungerbühler K (2013) Use patterns of leave-on personal care products among Swiss-German children, adolescents, and adults. Int J Environ Res Public Health 10:2778-2798. https://doi.org/10.3390/ijerph10072778

36. Korean Statistical Information Service. Summary of people, family, and house. https://kosis.kr/statHtml/statHtml.do?orgld=101\&tblld =DT_1IN1502\&conn_path=12. Accessed 25 Apr 2019

37. Korean Statistical Information Service. Summary of family by size of family. https://kosis.kr/statHtml/statHtml.do?orgld=101\&tblld=DT_1JC15 02\&conn_path=12. Accessed 25 Apr 2019

38. Korean Statistical Information Service. Summary of population by age and sex. https://kosis.kr/statHtml/statHtml.do?orgld=101\&tblld =DT_1IN1503\&conn_path=12. Accessed 25 Apr 2019

39. SCCS (2018) The SCCS notes of guidance for the testing of cosmetic ingredients and their safety evaluation, 10th revision, in SCCS Notes of Guidance. Publications Office of the European Union, Luxembourg. https ://doi.org/10.2875/77673

40. Korean Statistical Information Service. Summary of internet usage by age. https://kosis.kr/statHtml/statHtml.do?orgld=127\&tblld=DT_MH001 _Ml003\&conn_path=12. Accessed 13 June 2019

\section{Publisher's Note}

Springer Nature remains neutral with regard to jurisdictional claims in published maps and institutional affiliations.

\section{Submit your manuscript to a SpringerOpen ${ }^{\odot}$ journal and benefit from:}

- Convenient online submission

- Rigorous peer review

- Open access: articles freely available online

- High visibility within the field

- Retaining the copyright to your article

Submit your next manuscript at $\boldsymbol{\nabla}$ springeropen.com 